\title{
Challenges of teaching Kiswahili polysems and homonyms through translation in foreign language classes
}

\author{
Pendo Salu Malangwa ${ }^{1}$ \\ University of Dar es Salaam, Tanzania
}

\begin{abstract}
In teaching a foreign language, certain language features (i.e. polysems and homonyms) are not introduced as issues or topics for discussion and therefore, they emerge as weeds in the discussion (Klepousniotou, 2002). When this occurs, instructors struggle to handle them differently. This article investigated the challenges of teaching Kiswahili polysems and homonyms through translation in foreign language classes. The data for this study was collected through observation and documentary review methods. Findings show that Kiswahili has a complex system of polysems than homonyms. It has been further observed that translation method alone may not be adequate in handling problematic issues such as polysems and homonyms. Since polysems and homonyms are characterized by multiple meanings, a combination of translation method and componential analysis (analysis of semantic features) works better. Lastly, instructors should teach them in context instead of treating them as isolated words and they should be introduced at the intermediate through advanced levels of foreign language proficiency.
\end{abstract}

\section{Introduction}

Polysems and homonyms exist in almost all languages. However, as Klepousniotou (2002) argues, when teaching any foreign language ( $F L)$, polysems and homonyms are not introduced as issues or topics for discussion. They just come up as weeds in the teaching and learning process. In most cases, it happens when the learners are struggling to get a one to one correspondence between a foreign language word and their language. Unlike native speakers who do not use the language consciously, non-native speakers encounter difficulties in learning the words with multiple meaning (Koskela \& Murphy, 2006). This becomes more evident when translation method ${ }^{2}$ is applied to teach a foreign language. As it is with other language experts, like lexicographers, who are forced to list with numbers all possible meanings for polysems and homonyms, foreign language instructors are forced to list all possible meanings along with their translation equivalents. Translation method for a long time has been used to assist foreign language learners to understand the language and culture of the target language (TL) through their mother tongues. Stern (1992) argues that translation in one way or another can play a central

\footnotetext{
${ }_{1}^{1}$ Pendo Salu Malangwa is a senior lecturer and Director of Quality Assurance at the University of Dar es Salaam. She teaches in the Department of Kiswahili Language and Linguistics, Institute of Kiswahili Studies of the University of Dar es Salaam. She holds M.A.and PhD (Linguistics) from University of Dar es Salaam.Her areas of specialization and research interest include Translation, Interpreting, Terminology, Semantics and Pragmatics and has authored several papers in her areas of specialization. Email: pmalangwa@yahoo.co.uk.
}

2 Translation method (sometimes known as Grammar translation method) is a method of foreign language teaching which makes use of translation and grammar study as the main teaching and learning activities. (Gina, H. (2017) Grammar Translation Method. Retrieved January 20, 2010, from https://prezi.com/ugthoudkgrzp/grammar-translation-method/. 
role in foreign language teaching and learning processes. This was supported by Mwansoko et al (2006) who argue that translation has played a great role in helping students to learn a new language. Through translation, they can learn the differences and similarities between their mother tongue and the new language. A simultaneous understanding of the two languages could actually make the students to see the point of convergence and divergence more clearly. Damiani (2003) argued before that as opposed to other methods which can limit the teacher to use simple and familiar phrases, translation method gives the teacher freedom to use difficult and new vocabulary and expressions by giving the students their equivalents in TL. This helps the students to learn more in a short time.

Although the application of translation as a method for teaching foreign languages sounds smooth in terms of the transfer process, it has been criticised to have certain challenges (Malangwa, 2012; Chang, 2011; Damiani, 2003 \& Murphy, 2000). Translation as a practice itself is a mediation between two cultures. Kramsch (2004) argues that there is a connection between language and identity of a social group (i.e. culture). There are words and phrases in one language or another which are closely connected with the cultural contexts. Translating them into another language creates challenges of lacking equivalent forms and concepts in the destination language (See also Gee \& Lankshear, 1996). McKay (2002) argued that to use a language for special purposes, one needs is to learn the culture associated with the aspects of the discourse. Thus, the teachertranslator needs to know the concept of the words and the contexts which they are used, if he or she wants to be precise. Another question that may add value in the discussion relates to the issues of ambiguous forms like polysems and homonyms. What happens in the context of foreign language classes when they encounter polysemous words and homonyms in the course of discussion or classroom interaction? Are the instructors prepared to handle the situation? Do the training manuals cover such issues? This article investigates the challenges of teaching Kiswahili polysems and homonyms through translation in foreign language classes.

\section{Literature Review and Theoretical Justification of the Study Why Polysems and Homonyms in the Discussion of Translation Method?}

Polysems and homonyms can be examined from different perspectives depending on the range of interests of the researcher. They can be examined diachronically or synchronically either in relation to the field of theoretical linguistics or to that of applied linguistics (Rashidi, 2013). In the present study, the analysis of polysems and homonyms is to a larger extent focused on applied linguistics (i.e. translation and foreign language acquisition). Some modern linguists use the term polysemy to refer specifically to multiple meanings of words in which meanings are related. Homonyms, on the other hand, are defined as words that have the same form but unrelated meanings. The term ambiguous words has also been used to describe words that are either polysemous or homonymous in nature. Polysems and homonyms are all classified as different variations of lexical ambiguity (Clemmons, 2008). Rashidi (2013) maintains that polysems and homonyms are challenging issues for theories of semantic representation, semantic compositionality, language processing, machine translation and communication. While polysemy is the ambiguity between various related senses of a lexeme, homonymy is the ambiguity between completely unrelated or diachronically separate meanings. For that matter, teaching them has been a challenge in language classes and more challenging in foreign language classes.

Richards and Rodgers (2001) assert that due to the fact that language features such as polysems are the core of using a word form to refer to more than one meaning, its handling possess a great challenge to fields such as lexicography and translation studies. These kinds of words with multiple meanings always run the risks of misinterpretation and can easily result in ambiguity, which is mainly resolved through contexts and the available body of knowledge gained by humans. That means, using a translation method to teach polysems and homonyms to foreign 
language learners who are not conversant with the context of their use may not be suitable. The translation method may work to those who have mastered the language to the extent that they can read and understand the language like native speakers of the language (i.e. advanced language learners and somehow those at the intermediate level).

Singleton and Ryan (2004) believe that by supplying the learners of a foreign language with a cognitive motivation for the multiple meaning words, the teaching and learning of vocabulary is facilitated. The learners' awareness of the underlying meaning (basic or core meaning) of a word will strengthen their ability to retain and recall the vocabulary. The assumption that Singleton and Ryan are trying to bring here is that polysems and homonyms should not be introduced to foreign language class of the beginners. They should come later in the course of learning whereas the level of proficiency is developed to the extent of near native speakers. For the new language learners, it is easier for them to learn, remember and use something that is motivated than something that is arbitrary (Touplikioti, 2007).

When compared to homonymy, polysemy is considered as a frequently occurring language universal feature. It is central to language and cannot be ignored in the language teaching and learning processes. Clemmons (2008) emphasizes that polysems and homonyms do not occur by chance, they are caused by a human need to structure experience, knowledge and language. Due to this fact, it is always difficult to find corresponding equivalents in another target language. Both polysems and homonyms imply difference in meaning and formal identity. They both depend on context to a large extent for successful processing. For that matter, in the foreign language class they should be introduced only when the learner is able to use the language and differentiate the contexts of its use. However, they will always pose a challenge when using a translation method in teaching a foreign language, which is the central issue for the current study.

\section{Introducing Multiple Meaning Forms in a FL Class}

Vocabulary and basic phrases in a foreign language class are key issues in all comprehension. However, sometimes both instructors and students do not anticipate the obstacles that could be created by forms that have multiple meanings (like homonyms and polysems). Most training manuals also do not adequately take into account the high level of polysemy of many natural language words and phrases (Clemmons, 2008). As said in the previous section above, Touplikioti (2007) argues that human language users have a natural propensity for making polysemous extensions that lead to the increased use of certain meanings. This makes it easier for them to learn, to remember and even to use something that is motivated. For the second or foreign language learners, they are struggling to learn most of the things in an arbitrary situation, even for the meanings that were motivated. Instructors should be alert of the situation in order to make the learning happen in a more desired manner.

Aitchison (1994) as quoted in Rashidi (2013) argues that learning new vocabulary is not equivalent to simply attaching new meanings to word forms. It is a more complicated task. Aitchison stated three different tasks with which children acquire their first language (natural language) and which should be taken into consideration while teaching a foreign language. The first task is the labeling task during which the child attaches a label to a particular concept or object. The second task is the packaging task, in which the child has to learn the exact extension of the meaning of words. In this task, the child learns a category of objects that can be referred to by a single label; here the issue of multiple meanings is accommodated. The last stage is the task of network building, by means of which words would be fitted together in a semantic network. All these tasks happen in learning a second or foreign language as well. In the foreign language class, we have category of learners in terms of competence levels; beginners, intermediate and advanced learners (to be discussed later in this section). 
In learning a foreign language, the task for the beginners relates with the first task by Aitchison, labeling task and is performed in terms of associating with their first language. What they normally do is to search for equivalents between their first language and the foreign language that is introduced. At the intermediate level, the foreign language learners begin to learn the extension of the meaning of words (i.e. they begin learning extended meanings of the words in that language). For the advanced learners, since they begin to see the language in its natural context, they continue building semantic network between the words of the language. They understand and appreciate the semantic features of words in the foreign language and start lifting them together in a semantic network. By doing so, they are consciously or sub-consciously learning that the language has words that are synonymic, homonym, polysemous and antonym in nature. Those who are introducing learners into a second or foreign language, they have to be aware of what transpires the learning process at different levels of proficiency. Semantic issues should be introduced carefully and it should begin at intermediate level and proceed more and more at advanced level.

Dufour and Kroll (1995) argue that in fluent foreign language learners the connection between equivalent words of the first and foreign language is through concept (meaning) mediation or association between the words of two languages. As for Dufour and Kroll (1995), there are two hypotheses regarding the association between words of their first language and those of the new language in general: concept mediation and word association. The word association hypothesis posits that a direct association exists between words in the two languages, whereas the concept mediation hypothesis proposes that the only connection between the two languages is via an underlying conceptual system. Word association claims that for a language learner to produce or comprehend words in a second or foreign language, first he/she has to learn the underlying meaning of the word and retrieve its equivalent in his/her first language. In addition, Potter, So, Von Eckardt and Feldman (2004) concluded that more fluent language learners who can directly access meanings for their second language through their conceptual networks, act faster in lexical decision tasks, while less-fluent bilinguals are slower. They argued that less fluent bilinguals are able to access limited conceptual information from the second language, and are gradually progressing towards the full access. This connotes that complex language features should be introduced slowly in the foreign language learning process and after the learners have mastered most of the basic features.

As Rashidi (2013) argues, vocabulary or basic phrases senses are better acquired when encountered in the context of use. Teaching only sense selection in isolated forms might lead to teaching many exceptions and this hinders students' generalization and leads to creating learning hindrance. Firth (1957) claimed that we know a word by the company it keeps. This emphasizes the fact that, semantic complex issues like polysems and homonyms should be introduced at a more advanced level of language proficiency. Rashidi (2013) further contends that it is more sensible to provide foreign language learners having low competence in that particular foreign language with abstract underlying meanings as an appropriate starter. This can help them in their advanced stage to acquire multiple meanings of words in the future. When complex issues are introduced at the basic level, it may hinder the learning process and demotivate the learners from progressing.

\section{Categories of FL Learners and the Teaching of Polysems and Homonyms}

The challenges of teaching polysemy and homonymy in a foreign language class can be associated with the level of foreign language proficiency of the learners. Foreign language learners can be divided into three groups based on their proficiency levels of language competence (i.e. based on the language learning evolution). There are beginners, intermediate 
and advanced learners (Brown, 1994). The beginners are usually so much excited to learn the basic issues in the foreign language, such as aspects related to greetings, introducing one another, the basic vocabulary of food and drinks, knowing directions as well as family relationship. Brown (1994) emphasises that at this level, the learners' interest is mainly to establish a relationship between their language and the new language they are trying to learn. To them everything is new and it takes time to get used to the new language vocabulary, sounds, patterns, grammatical structures and rhythm. The brain takes so much time to process the quantity of new information that is being exposed to. Instructors at this level should be very careful not to introduce issues that may frustrate the learning process. All complex morphological, syntactic, semantic and stylistic issues should await for the next levels.

The intermediate learners are always interested to see the language in context. At this stage, things are getting easier for them and the language learning no longer sounds so foreign. The learners are able to recognize the rhythm of the language and pick out its sounds easily. When a native speaks about familiar topics, the learners can understand the bigger picture of what is being said and can identify speech patterns in the language. Generally, acquiring sounds, words and structures become quicker and easier. At this level, they may begin learning few complex semantic issues by associating with what they learnt at beginners' level (underlying meaning). The advanced learners are expected to be moving to more advanced skills of a foreign language and actually, they are curious to learn the language in context. Instructors should be in a position to see if they can learn complex features of the language. The advanced learners explore complex issues like idiomatic and literary expressions, technical language as well as sense relations. They can understand native speakers, follow TV show and can read newspapers easily ${ }^{3}$. This implies, while the instructor is struggling to find the best ways to introduced them to complex features of the language because they have already mastered the basics of the language and now they can read. The role of the teacher here is to guide them on how best they can learn and retain for future use.

Leyre (2014) argues that progression between these three levels is not strictly cumulative. Most students are at what he calls an intermediate stage, which is also the difficult stage to teach because the learners now feel they have acquired a lot and they can understand and follow easily. While this argument is true, the level of competence between intermediate and advanced in some cases is cumulative. The advanced students are autonomous language learners; they can progress on their own without a teacher or structured method, through sheer immersion, while intermediate learners still require scaffolding from a teacher, a book, or any structured learning system. In that regard, the levels or categories of the learners should not be ignored while discussing complex language features in the process of teaching a foreign language.

The Institute of Kiswahili Studies of the University of Dar es Salaam receives almost all of the three categories, but with a limited number of beginners (Rubanza, 2012). While enjoying the learning process in the natural setting, students interact with Swahili speakers and bring in their classroom interaction any difficult or complex issues they have come across in the course of their interaction with native speakers. Apart from the common vocabulary, they bring complex issues like idiomatic expressions, sayings, synonyms, homonyms, polysems, contrast and they want to get answers from their instructors. As for Leyre (2014), language learning requires three very different activities, especially for intermediate and advanced:

\footnotetext{
${ }^{3}$ See https://www.lucalampariello.com/the-3-stages-of-language-learning-evolution/, accessed on 21/01/2019 at 6:00AM.
} 
1. Growth - Here students work hard to learn new words or refine the meaning of known words, learn more advanced grammatical patterns, improve pronunciation accuracy, and develop more adapted rhetorical skills.

2. Clarification - Students work hard to correct errors in pronunciation, grammar, use of words; fill up gaps in certain semantic or pragmatic areas. By doing so, the learner understands and corrects recurring errors. This clarification phase could also be called 'systemic integration', as what needs to happen is a fast check of the whole system every time an error is detected.

3. Endurance - Automate whatever is known already: the same task should be done with less effort and more quickly, with more distractions present, or in a more stressful setting.

Instructors are required to be smarter in handling such situations in order to facilitate the learning process the second or foreign language class.

\section{Methodology}

This study was carried out at the Institute of Kiswahili Studies of the University of Dar es Salaam in Tanzania, which represents units that receive Kiswahili foreign language learners almost every year from America, Asia and Europe. In addition, the Institute has a long history of teaching Kiswahili to foreigners compared to other institutions in the East Africa region and beyond. The data for this study was collected through observation and documentary review methods. In applying the observation method, the researcher observed 5 randomly selected Swahili advanced and intermediate classes whereby in one of the classes the researcher participated or was involved in the teaching process to assist the main instructor. This helped the researcher to learn the challenges of teaching polysems and homonyms and consequently decided the write about this research topic. The researcher used classes for intermediate and advanced learners (and of course as noted early, the Institute of Kiswahili Studies receive many students from these levels) because of the nature of the study as the issues of polysems and homonyms emerge in such levels. So, through observation, the researcher managed to collect 5 polysems and 5 homonyms that were clearly introduced and the learners indicated an interest to learn more in the next classes.

In the documentary review, the researcher reviewed two training manuals used in teaching Kiswahili to foreigners, namely Sema Kiswahili: Furahia Tanzania by Peace Corps Tanzania and Kiswahili Hazina ya Afrika: Kitabu cha Kwanza by Yunus I. Rubanza, Aldin K. Mutembei, Titus Mpemba and Elizeus G. Katikiro. The two manuals were selected on the basis that one was written by Tanzanians in collaboration with Americans and the other one was written by Tanzanians, and therefore, they can give a good sample for the study. The two books present Kiswahili vocabulary and expressions along with their English translations. In additions, the researcher had also an opportunity to review some training handouts that were presented by the instructors to the students to aid their discussions on polysems and synonyms. This was mostly observed in the classes for advanced learners whereby they were presented with newspapers, poems and cartoons for discussion. Through the documentary review, only 3 Kiswahili polysems and 2 homonyms were collected in line with their English translations. However, a closer analysis of the data that were collected through observation and documentary review methods indicated that some of the data used in the classroom interactions were the ones used the documents reviewed. That means, only a total of 6 polysems and 4 homonyms were collected through the two methods and the data enabled the researcher to draw conclusions and generalizations in relation to the research topic.

The data for this study has been analyzed descriptively and in a comparative way with their English equivalents. In other words, the Kiswahili expressions are presented along with their 
English translations and in a more descriptive approach. Generally, the two data collection methods were very useful and complementary in obtaining the data for this study.

\section{Findings \\ Challenges in Teaching Kiswahili polysems and homonyms}

The researcher reviewed two Kiswahili training manuals and handouts used in the teaching process and observed some classroom interactions in 5 classes. As noted earlier, the researcher managed to collect a total of 6 polysems (whereby it was established that 2 were nouns and 4 verbs) and 4 homonyms (2 nouns and 2 verbs) for analysis. These forms were repeatedly presented in the classes observed and even in the training manuals. This is due to the reasons that, polysems and homonyms were not introduced as topics for discussion, but rather in the readings they came across such words and accelerated their discussion to bring some addition forms. This is in line with the claims by Klepousniotou (2002) who argue that polysems and homonyms are not introduced as issues or topics for discussion. They just emerge as weeds in the classroom interactions.

It was further observed that while the documents reviewed employed just the translation method, classroom interactions used translation method as well as componential analysis methods. Componential analysis methods refers to analysis of meanings or semantic features entailed in the various forms of the language and in this case, Kiswahili polysems and homonyms as presented in the subsections below. Furthermore, in studying the data it was evident that Kiswahili, like many other languages, has a complex system of polysems than homonyms (Firth, 1957). That means, while the meanings in homonyms can be exhaustively listed, meanings in polysems are unlimited. For instance, most of the polysemous verbs pick nouns easily and become more idiomatic. In that way, they become a challenge while translating for foreign language learners enable them to acquire and use such structures or vocabulary properly. On the contrary, homonyms have limited or fewer meanings, although some homonyms were also to have features of polysems. That implies, they are both homonyms and polysems. The following subsections are discussing the two aspects separately for the aim of drawing generalizations and conclusion.

\section{Challenges of Teaching Polysems}

In studying the challenges related to polysems, it was observed that although the literature claims that meanings of a polysemous word are related but their translations in the classroom interactions which used more of the communicative translations proved the opposite. It was evident that they are unrelated. Moreover, although the meanings in such words are unlimited, instructors concentrated on the commonly used senses which helped the group to be more focused. Instructors were giving a portion of meanings by ways of listing in order to avoid confusing the students. It was further observed that the students continued to learn other extended meanings on their own (out of the class) but they brought to the class for the instructors to assist with meanings of translations. In one of the classes, a student brought a Swahili word (Jipu-Boil) that she heard people mentioning it several times and she also heard that same word in public transport, then she wanted to know the meaning of that word in English. When she asked in the class, the instructor first gave the underlying meaning together with its English equivalent and thereafter, the teacher continued to list some contextual meanings (which were actually more political as the word has currently acquired political related meanings) as noted below:

A: Jipu (Boil)

(1) Uvimbe wa kwenye ngozi (a painful nodule formed in the skin/skin inflammation)

(2) Siri (top secret) 
(3) Mtu mbaya (Corrupt person)

(4) Muuza madawa ya kulevya (drug dealer)

(5) Mtu mzembe (Irresponsible/lazy person), etc.

The instructor added that "in the context of Tanzania, since 2015 the word Jipu is so much used all over the country" and promised to bring some cartoons that may help to understand these concepts in the next class. In the following class, the instructor brought the following cartoons for more elaboration and the students were asked to give interpretation (since now they have the idea):

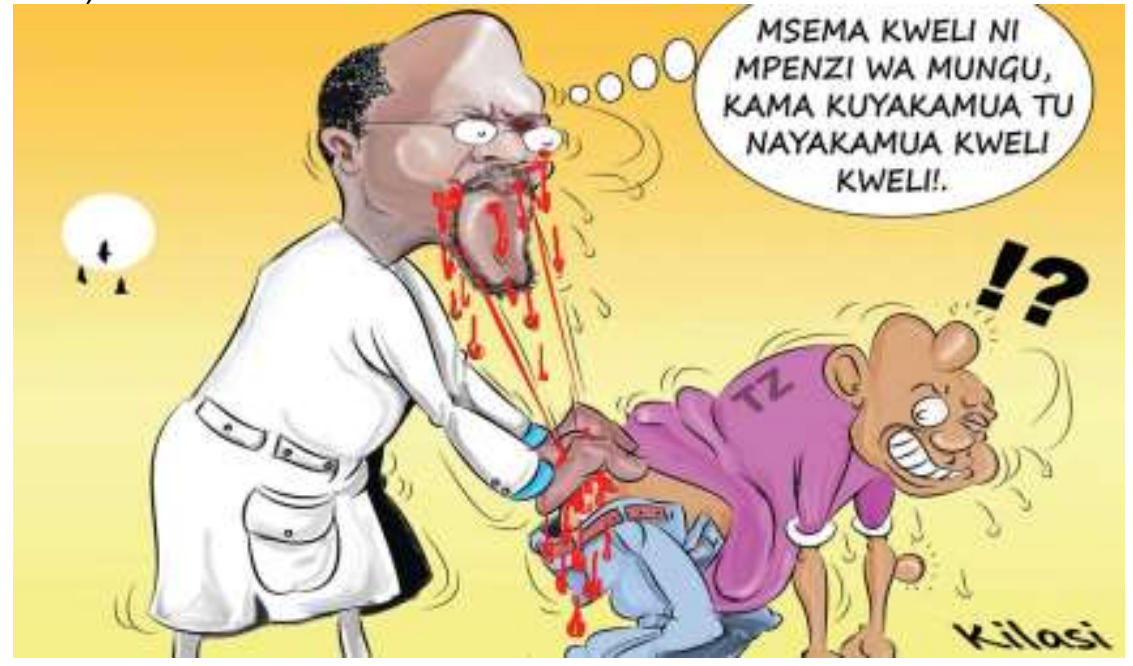

Figure 1. Cartoon of H.E. President Magufuli Tumbling Boil

The instructors helped the students understand the word jipu using this cartoon. It was elaborated that "when President Magufuli entered in power, Tanzania had a lot of majipu (problems and corrupt people). Generally, the word jipu has been associated with a number of corrupt behaviours in the society, and for President Magufuli all these are "majipu" (boils). When a top secret or underground issue (i.e. corrupt movements) is disclosed, they say; Jipu limetumbuliwa (boil had been tumbled). The list of meanings in the word Jipu is now very long". To make the point more clearly on how the list of associated meanings is long, the following cartoon was distributed to the students facilitate the learning of the meanings associated with the word jipu:

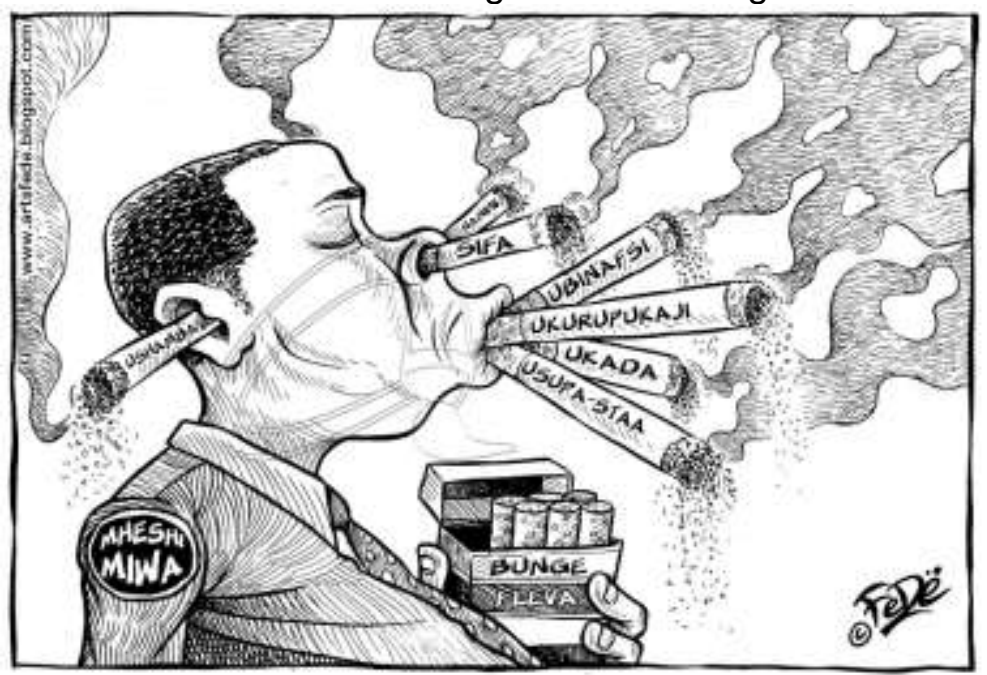


Figure 2. Cartoon Showing Various Boils a Person may have (H.E. Member of Parliament)

The instructors continued to clarify that this cartoon is representing a Member of Parliament who has a number of boils ranging from USHAMBA (hooligan/lout) to USUPA-STAA (super star) behaviors. From the above illustrations, it is evident that the word Jipu was alarming to the foreign language students in the context of language use. Its use was very broad and there was a need to understand its meanings. However, the translation method alone could not handle the situation. As observed, the instructor used certain illustrations and context-based examples for them to learn the multiple senses easily. The instructors from this class added that in Kiswahili, there are many words that have associative meanings and their interpretations require contexts. He said; "if you hear a word like endesha (drive), it may refer to fanya gari lisogee kutoka sehemu $A$ kwenda $B$ (moving a car from point $A$ to $B$ ), fanyia mtu mambo ya kuudhi (ill-treat one), harisha (someone is experiencing diarrhea), etc".

The problems related to unlimited meanings (of polysems) were also observed in another class for the words like Kichwa and the instructor was trying to list the meanings along with their translations as observed below:

\section{B: Kichwa}

(1) Sehemu ya mwili (head-part of a body, leader or bright person.

(2) Kiongozi wa familia (head of family)

(2) Sehemu ya mbele ya treni (train engine)

(3) Kifuniko cha chupa (Bottle top)

(4) Mada ya habari (News headline), etc.

Through observation in the examples that were used in class (with also the handouts supplied to the learners) and the data from internet sources that are used to teach Kiswahili to foreigners, it was evident that the problem of polysemous words is more critical with the words in the verb class. Apart from the meanings entailed in polysemous verbs, this category of words picks nouns easily and create meanings that look sometimes completely unrelated. See for example the verb Kata that was used in a class of advanced learners when they were accidentally (since it was not a topic for that day) introduced to polysemous words in one of their classes:

C: Kata

(1) Kata (to divide into portions)

(2) Kata rufaa (to appeal)

(3) Kata tiketi (buy a ticket)

(4) Kata kiuno (dancing)

(5) Kata kamba (die)

(6) Kata kiu (quench thirsty), etc.

From these examples, it can be clearly observed that the meanings generated after acquiring a noun are highly idiomatic. They lack semantic relationship with the basic meaning of the verb Kukata (to cut). While observing interactions in the following class, the instructor elaborated that one Poet, M. Khatibu) decided to show that some words in Kiswahili are very rich in meanings and cited one poem from Khatibu $(2007$, p. 8):

D: $\quad$ Kata, ni kulikata kuti, kwa panga au kitara Kata, ni kunjiko la kati, umbo la nyoka duwara

Kata, ni kujitwika mti, unajiwekea tambara

Kata, ni kuponya mauti, kiu inapocharura 


\section{Mgodi wa Kiswahili ni dafina isokwisha}

From this poem, the instructor highlighted that through this poem/stanza the word Kata belongs to both verb and noun classes. It has different meaning in each verse and the stanza concludes by saying that Kiswahili is like a gold mine, you can't exhaust, which implies that words in Kiswahili acquire meanings daily. Moreover, in about three (3) foreign language classes, when the instructors were trying to list the meaning of one polysem, students raised other meanings of those same words in the context they are used other than those which were listed in the previous class. Students were observed to have a lot of confusion in learning and using such words. As with Kata, the Kiswahili word Piga was also observed from the training manuals and the internet sources to have features of polysems but sometimes the meanings are not closely related for the learners to associate as seen below:

\section{E: Piga}

(1) Piga picha (take a photo)

(2) Piga mtoto (bit a child)

(3) Piga mbizi (dive)

(4) Piga hodi (nock)

These four uses (meanings) of piga were highlighted in one class and in the following class students came with the following more expressions involving the piga (some of their translations were provided in the class).

F: (5) Piga magoti (kneel down)

(6) Piga chafya (sneezing)

(7) Piga kelele (make noise)

The instructor gave English equivalents as seen above and after giving the equivalent translations; however, the learners could not see any match with the examples in $E(1-4)$. The instructors clarified that these were somehow figurative, since their meanings are idiomatic. This also emerged in the discussion on the multiple meanings in fuga (keep anima or bird at home). In the two verbs other idiomatic meanings include fuga ndevu (literally staying with beards for a long time with cutting them) and fuga ujinga (literally staying with ignorance). The literal translations here were a bit more meaningful and somehow were closely relating with the underlying meaning.

These were discussed one after another by providing translations after explaining their contextual use. This actually situation interrupted the learning process and students started to struggle with listing the different meanings for one word by using dictionaries and sometimes asked for interpretation. Some instructors thought it was an easy work but they ended up getting tired and asked students to ignore some of the meanings because they were not common. In fact, polysemous expressions are more idiomatic and they change radically from the basic meaning of the word. Scholars of semantics, like Leech (1974), claim that unlike homonyms, the meanings in polysemy are related. However, when teaching polysemy through translation, it is evident that they are not so much related. It requires the instructor to give more elaborations using both linguistic and non-linguistic illustrations as observed in the classes that were used for investigation.

\section{Challenges of Teaching Homonyms}

This section presents the data that was collected in relation to homonyms. In the teaching process, it was observed that while using the translation method, the instructors of almost all 5 classes were forced to structurally reconstruct the word ota to facilitate the learning. Fortunately, 
they were all linguists and therefore, they all have an idea what happened for the three words to have one form. See the following analysis that was given for this case:

E: Ota -

(1) Ota - to dream originally lota

(2) Ota - to germinate originally ota

(3) Ota - to warm originally kota

While teaching the meaning of the word ota, the instructors were showing step by step how sounds $/ \mathrm{l} /$ and $/ \mathrm{k} /$ were dropped over the history and finally we have three words in Kiswahili that are morphologically related but semantically unrelated. The analysis here was so much complicated for them to comprehend but they were forced to memorize them in terms of their semantic features and the context of their use. This analysis was unique when compared to other homonyms that were observed in some of the training materials for the words panda and anuani, including the following:

F: Panda -

(1) Panda - to climb

(2) Panda - forehead

(3) Panda - Junction

G: Anuani -

(1) Anuani - Adress

(2) Anuani - Theme or title name of the book

It was further clarified that for some words like panda and anuani above, their morphological resemblance just occurred by coincidence. That means, there is no historical explanations as to why there is such a situation in the Kiswahili language. So, the learners were also encouraged to memorize their meanings and/ or the equivalents in English but it looked difficult. In another class, when the instructor was teaching a topic on Family, and with the understanding that the learners were advanced, he was tempted to introduce other homonymic senses for the word babu as seen below:

$\mathrm{H}: \mathrm{Babu}-$

(1) Babu - a male parent to mother or father

(2) Babu - a male that advises women group

(3) Babu - a disease affecting children at their young age that resembles epilepsy

(4) Babu - a tool that measures weight or length of a cloth/garment

(5) Babu - Part/Section in a book that is complete

After the instructor listed the meanings (translations) of the word babu, he further went to indicate the commonly used homonyms. It was illustrated that Swahili speakers commonly use the senses in $\mathrm{H}(1)$ and somehow $\mathrm{H}(3)$. The analysis of the data on homonyms indicate that meanings in homonyms are unrelated and the instructors taught them as independent words. However, the translations given by instructors on the uncommonly used words are difficult to grasp and unlike in polysemy, the instructor here could not provide illustrations but rather encouraged students to ignore them. Klepousniotou (2002) argues that teaching the forms of language which are homonymic or polysemous in nature through translation method is challenging and for that matter, a combination of methods would help in facilitating the learning process. 
In addition, given that most of the meanings in these words are more of an idiomatic, the instructors use various strategies to handle the situation. The first approach that was mentioned by the instructors to be used in assisting the student is the use of literal translation. When translating the above polysems and homonyms communicatively, they do not make sense to the students because the meanings provided do not relate to the basic or underlying meaning of that word. As a way to assist the learner, the instructors usually resorted to translating them literally. Most instructors also avoided teaching uncommon and archaic meanings or usages that are no more used in the language. This helped to minimize the complications in the learning process as the meanings became somehow minimal.

The third and last strategy that was listed to be used in resolving the above discussed challenges is the use of descriptive translations for certain concepts (Malangwa, 2012). The instructors in some classes resorted to providing descriptive translations accompanied by some signs/cartoons or pictures. This facilitated the learning process of polysemous vocabulary as well as the homonyms.

\section{Conclusion and Recommendations}

Several issues have been observed through this study with regard the challenges in teaching polysems and homonyms to foreign language learners through the translation method. As noted above, it is evident that although languages have a complex system of polysems, both polysems and homonyms are complex and require careful handling while introducing them to foreign language learners. It always requires the instructor to devise some methods and strategies to facilitate learning. Among the methods and strategies, it is advisable to include application of literal translation, retrieving the etymology of the words, as well as avoiding uncommon meanings or archaic use of the word. Through this study it is suggested that for both polysems and homonyms:

(i) In connection with the translation method, instructors should teach polysems and homonyms in context or in collocation instead of isolated words;

(ii) instead of using communicative approaches to translate the forms or expressions, instructors should opt for literal translation methods which helps the learners to see the correlation between the underlying meaning and the extended meanings in polysems; and

(iii) students should be taught to accept that they should be prepared to accept the fact that many words in many languages have multiple meanings. Their first language can be used to facilitate this.

Generally, the challenges of teaching polysems and homonyms are not new to language instructors. In a foreign language class instructors need a combination of approaches to handle such expressions (like a combination of translation method and componential analysis or analysis of semantic features). Lastly, polysems and homonyms should be introduced at the intermediate and advanced levels when language proficiency is well developed.

\section{References}

Aichison, J. (1994). Words in the Mind: An Introduction to the Mental Lexicon. Oxford: Blackwell. Brown, H. H. (1994). Principles of Language Learning and Teaching. Englewood Cliffs, NJ: Prentice Hall Regents.

Clemmons, K. (2008). The Problem of Polysemy in the First Thousand Words of the Generak

Service List: A Corpus Study of Secondary Chemistry Texts, Unpublished Doctoral

Dissertation, University of Central Florida. 
Chang, S. (2011). A constructive study of grammar translation method and communicative approach in teaching English grammar. English Language Teaching, 4(2), 13-24.

Damiani, A. J. (2003). The grammar translation method of teaching. London: Longman.

Dufour, R., Kroll, J. F. (1995). Matching words to concepts in two languages: A test of the concept mediation model of bilingual representation. Memory and Cognition, 23(2), 166180.

Firth. J. R. (1957). A synopsis of linguistic theory 1930-1955. Studies in Linguistic Analysis, 132.

Gee, J. P., Hull, G., \& Lankshear, C. (1996). Socio-cultural literacy, discourses and the new work order. The New Work Order: Behind the language of New Capitalism, 1-23.

Khatibu, S. M. (2007). Fungate ya Uhuru, Dar es Salaam: Macmillan Aidan Ltd.

Klepousniotou, E. (2002). The Processing of Lexical Ambinguity: Homonymy and Polysemy in the Metal Lexicon. Brain and Language, 81(1-3), 205-223.

Kramsch, C. (2001). Language and Culture. Oxford: Oxford University Press.

Koskela, A. and Murphy, M.L. (2006). Polysemy and homonymy. Encyclopedia of Language and Linguistics, 742-744.

Leyre, J. (2014, January 27). Language Levels-Beginner, Intermediate, Advanced, Retrieved from https://julienleyre.me/2014/01/27/language-levels-beginner-intermediate-advanced/.

Malangwa, P. S. (2012). Overcoming the barriers through literal and descriptive translations: Examples of Kanga names. Journal for Studies in Humanities and Social Sciences, 1(1), 5162.

McKay, S. (2003). The cultural basis of teaching English as an international language. TESOL Matters, 13(4), 1-4.

Murphy, R. (2000). German in Use: Self-study reference and practice for students of English. Cambrige: Cambridge University Press.

Mwansoko, H. J. M, Mekacha, R. D. K., Masoko, D. L. W. \& Mtesigwa, P. C. K. (2006). Kitangulizi cha Tafsiri: Nadharia na Mbinu. Dar es Salaam: TUKI.

Nakahara, A. (2005). The effect of instruction of underlying meaning of polysemous words. Ph.D. dissertation, Retrieved from ProQuest (UMI No: 3178817).

Potter, M.C, So, K. F., Von Eckardt, B. \& Feldman, L. B. (2004). Lexical and conceptual representation in beginning and proficient bilinguals. Journal of Verbal Behavior, 23(1), 2338.

Rashidi, N. (2013). Teaching English polysemous words to Iranian EFL learners: Underlying meaning approach and selection in comparison. Journal of Language, Culture, and Translation (LCT), 2(1), 83-101.

Richards, C. J. \& Rodgers, S. T. (2001). Approaches and methods in language teaching. Cambridge: Cambridge University Press.

Rubanza, Y. I. (2012). Masuala ya Msingi katika Utayarishaji wa Majaribio kwa Wanafunzi Wanaojifunza Kiswahili kama Lugha Yao ya Pili. Mulika, 8, 48-64.

Singleton, D., \& Ryan, L. (2004). Language acquisition: The age factor. Clevedon: Multilingual Matters Ltd.

Stern, H. H. (1992). Issues and options in language teaching. Oxford: OUP.

Touplikioti, S. (2007). The teaching of polysemous verbs "make" and "do" to Greek learners of English: A Cognitive Linguistic Approach, Unpublished PhD Linguistics Thesis, Aristotle University of Thessaloniki. 